\title{
A Remark on a Necessary Condition of the Cauchy-Kowalevski Theorem
}

By

\author{
Keiichiro Kitagawa* and Takashi Sadamatsu*
}

\section{Introduction}

We are concerned with a necessary condition of the CauchyKowalevski theorem for the differential operator $L$ with the analytic coefficients in the neighbourhood of the origin:

$$
L=\partial_{t}^{m}-\sum_{j=1}^{m} a_{j}(t, x ; \partial) \partial_{t}^{m-j} .^{1)}
$$

Let

$$
a_{j}(t, x ; \partial) \equiv \sum_{\alpha} a_{j, \alpha}(t, x) \partial^{\alpha} \equiv \sum_{\alpha, \mu} a_{j, \alpha}^{\mu}(x) t^{\mu} \partial^{\alpha} \equiv \sum_{\mu} t^{\mu} a_{j}^{\mu}(x ; \partial)
$$

Professor S. Mizohata [3] defined the weight $q$ of $L$ by

$$
q \equiv \operatorname{Min}\left\{q ; \underset{\partial}{\operatorname{order}} a_{j}(t, x ; \partial) \leqq q \cdot j, \quad j=1,2, \ldots, m\right\}
$$

and, denoting by $h_{j}(t, x ; \partial)$ the homogeneous part of $a_{j}(t, x ; \partial)$ with order $q \cdot j$, he showed that, in order that the Cauchy-Kowalevski theorem for $L$ hold at the origin, it is necessary that

$$
h_{j}(0, x ; \partial) \equiv 0 \quad(j=1,2, \ldots, m), \quad \text { if } \quad q>1 .
$$

Mr. M. Miyake [2] investigates the first order operator $L^{1}$

$$
L^{1}=\partial_{t}-a(t, x ; \partial)
$$

Communicated by S. Matsuura, February 14, 1975.

* Department of Mathematics and of Applied Mathematics, Ehime University, Matsuyama.

1) We use the following abbreviations: $\partial^{\alpha}=\partial_{x}^{\alpha}, \partial_{t}^{j}$ stand for $\left(\frac{\partial}{\partial x}\right)^{\alpha},\left(\frac{\partial}{\partial t}\right)^{j}$ respectively. 
and proved that, in order that the Cauchy-Kowalevski theorem for $L^{1}$ hold at the origin, it is necessary and sufficient that

$$
\underset{\partial}{\operatorname{order}} a(t, x ; \partial) \leqq 1
$$

Professor S. Mizohata [4] investigates again a necessary condition of the Cauchy-Kowalevski theorem for $L$ and showed that it is necessary that

$$
p \leqq 1,
$$

where $p$ is the modified weight of $L$ which is defined by

$$
p=\operatorname{Min}\left\{p ;|\alpha|+p(m-j-\mu) \leqq p \cdot m, \quad a_{j, \alpha}^{\mu}(x) \not \equiv 0\right\},
$$

and that, in particular,

$$
\underset{\partial}{\operatorname{order}} a_{j}(0, x ; \partial) \leqq j \quad(j=1,2, \ldots, m) .
$$

Mrs. Y. Hasegawa [1] remarked that it is moreover necessary that

$$
\underset{\partial}{\operatorname{order}} a_{j}^{1}(x ; \partial) \leqq j \quad(j=1,2, \ldots, m),
$$

and sent us her manuscript.

In a series of these researches, they proved their results by means of the formal solution. Using their techniques, especially that of Mrs. Y. Hasegawa, we obtained the following result.

Let

$$
n(j, \alpha)=\operatorname{Min}\left\{\mu ; a_{j, \alpha}^{\mu}(x) \not \equiv 0\right\}
$$

Then the modified weight $p$ of $L$ is given by

$$
p=\operatorname{Max}_{j, \alpha}\left\{\frac{|\alpha|}{n(j, \alpha)+j}\right\}
$$

We define the modified principal part of $L$ by

$$
\sum_{j=1|\alpha|=p(n(j, \alpha)+j)}^{m} t^{n(j, \alpha)} a_{j, \alpha}^{n(j, \alpha)}(x) \partial^{\alpha} \partial_{t}^{m-j}
$$

and we say that the terms 


$$
a_{j, \alpha}^{\mu}(x) t^{\mu} \partial^{\alpha} \partial_{t}^{m-j} ; \text { for which }|\alpha| \leqq j
$$

belong to the kowalevskian part of $L$.

Theorem. In order that the Cauchy-Kowalevski theorem for $L$ hold at the origin, it is necessary that the modified principal part of $L$ is composed uniquely of the terms belonging to the kowalevskian part of $L$.

Let

$$
p_{k}=\operatorname{Max}\left\{\frac{|\alpha|}{n(j, \alpha)+j},|\alpha| \leqq j\right\}, p_{v}=\operatorname{Max}\left\{\frac{|\alpha|}{n(j, \alpha)+j},|\alpha|>j\right\}
$$

Then our theorem is also represented as follows.

In order that the Cauchy-Kowalevski theorem for $L$ hold at the origin, it is necessary that

$$
p_{v}<p_{k}
$$

Remarks. Suppose that the Cauchy-Kowalevski theorem for $L$ holds at the origin.

1) The theorem says that the $(j, \alpha)$ for which $p=\frac{|\alpha|}{n(j, \alpha)+j}$ satisfy $|\alpha| \leqq j$. This implies $p \leqq 1$.

2) In $a_{j}(t, x ; \partial)$ we observe the terms $a_{j}^{1}(x ; \partial)=\sum_{\alpha} a_{j, \alpha}^{1}(x) \partial^{\alpha}$. The theorem says that there does not exist the terms for which $|\alpha|=p(1+j)$ unless $p<1$. We have therefore $|\alpha|<1+j$, i.e. $|\alpha| \leqq j$. That is $\underset{\partial}{\operatorname{order}} a_{j}^{1}(x$; $\partial) \leqq j$.

\section{Formal Solution and Recurrence Formula}

We treat the following Cauchy problem;

$$
\left\{\begin{array}{l}
L(u)=n_{0} t^{n_{0}-1} f(x), \\
\left.u\right|_{t=0}=\cdots=\left.\partial_{t}^{m-1} u\right|_{t=0}=0 .
\end{array}\right.
$$

Setting $\vec{u}(t, x)={ }^{t}\left(u^{1}(t, x), \ldots, u^{m}(t, x)\right)={ }^{t}\left(u, \partial_{t} u, \ldots, \partial_{t}^{m-1} u\right)$, we have 
(2.2)

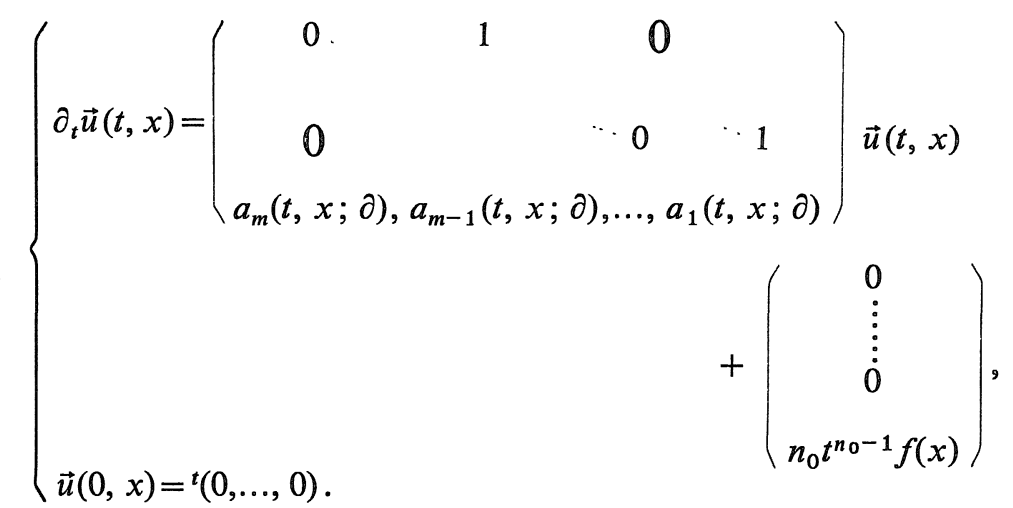

We denote the formal solution of (2.2) by $\vec{u}(t, x) \sim \sum_{n} t^{n} \vec{u}_{n}(x)=\sum_{n} t^{n} t\left(u_{n}^{1}(x)\right.$, $\left.\ldots, u_{n}^{m}(x)\right)$, then $\left\{\vec{u}_{n}(x)\right\}$ are determined by the recurrence formula:

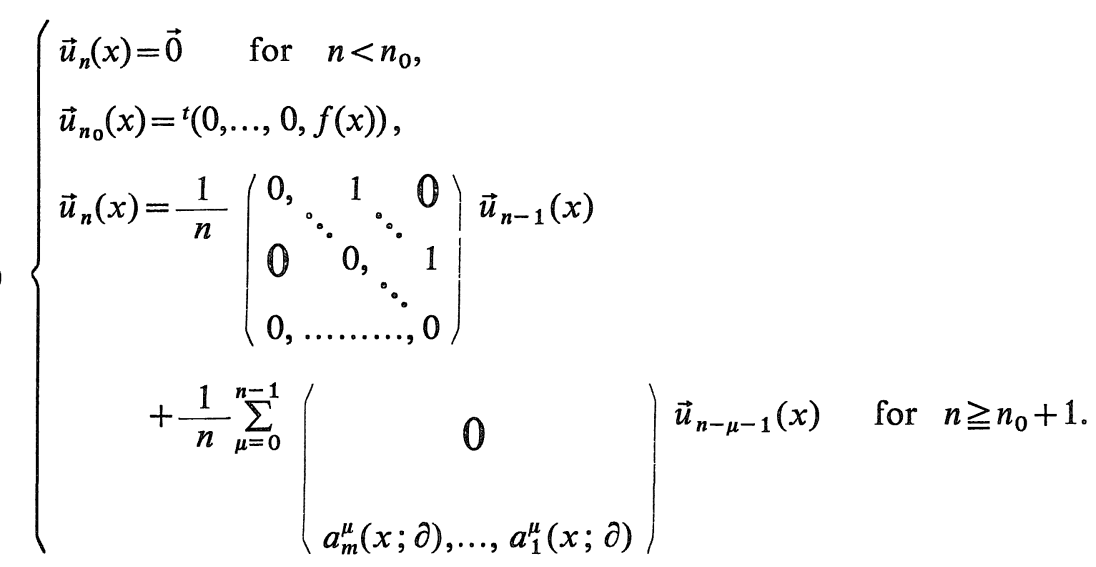

This also gives the recurrence formula for $\left\{u_{n}^{i}(x)\right\}$ :

$$
\left\{\begin{array}{l}
u_{n_{0}}^{i}(x)=0 \quad(i=1,2, \ldots, m-1), \quad u_{n_{0}}^{m}(x)=f(x) \\
u_{n}^{i}(x)=\frac{1}{n} u_{n-1}^{i+1}(x) \quad(i=1,2, \ldots, m-1) \\
u_{n}^{m}(x)=\frac{1}{n} \sum_{\mu=0}^{n-1} \sum_{j=1}^{m} a_{j}^{\mu}(x ; \partial) u_{n-\mu-1}^{m-j+1}(x) \quad \text { for } \quad n \geqq n_{0}+1
\end{array}\right.
$$

Let

$$
\sigma(j, n)= \begin{cases}1, & (j=1) \\ \frac{1}{n(n-1) \ldots(n-j+2)}, & (j=2,3, \ldots, m)\end{cases}
$$


then we have from (2.4)

$$
u_{n}^{m-j+1}(x)=\sigma(j, n) u_{n-j+1}^{m}(x)
$$

and

$$
u_{n}^{m}(x)=\frac{1}{n} \sum_{\mu=0}^{n-1} \sum_{j=1}^{m} \sigma(j, n-\mu-1) a_{j}^{\mu}(x ; \partial) u_{n-\mu-j}^{m}(x) .
$$

Denote briefly $u_{n}^{m}(x)$ by $v_{n}(x)$. Then (2.6) becomes

$$
\left\{\begin{aligned}
v_{n}(x) & =0 \quad \text { for } n \leqq n_{0}-1, \\
v_{n_{0}}(x) & =f(x) \\
v_{n}(x) & =\frac{1}{n} \sum_{\mu=0}^{n-1} \sum_{j=1}^{m} \sigma(j, n-\mu-1) a_{j}^{\mu}(x ; \partial) v_{n-\mu-j}(x) \quad \text { for } n \geqq n_{0}+1 \\
& =\frac{1}{n} \sum_{j=1}^{m} \sum_{\alpha} \sum_{\mu=n(j, \alpha)}^{n-1} \sigma(j, n-\mu-1) a_{j, \alpha}^{\mu}(x) \partial^{\alpha} v_{n-\mu-j}(x)
\end{aligned}\right.
$$

where we choose the number $n_{0}$ in such a way that $n_{0} \geqq \operatorname{Max}_{j, \alpha}\{n(j$, $\alpha)+1\}$ and that $p \cdot n_{0}$ is an integer.

\section{Lemma 1 .}

$$
v_{n}(x)=Q_{n}(x ; \partial) f(x)+R_{n}(x ; \partial) f(x) \quad \text { for } n \geqq n_{0},
$$

where (1) $Q_{n}(x ; \partial) \equiv 0$ for $n$ such that $p \cdot n$ is non integer,

(2) if $Q_{n}(x ; \partial) \not \equiv 0$, then $\underset{\partial}{\operatorname{order}} Q_{n}(x ; \partial)=p\left(n-n_{0}\right)$ for $n$ such that $p \cdot n$ is integer,

(3) $\operatorname{order} R_{n}(x ; \partial)<p\left(n-n_{0}\right)$.

Moreover $\left\{Q_{n}(x ; \partial)\right\}$ satisfy the following recurrence formula:

$$
\left\{\begin{array}{l}
Q_{n_{0}}(x ; \partial)=1 \\
Q_{n}(x ; \partial)=\frac{1}{n} \sum_{j=1|\alpha|=p(n(j, \alpha)+j)}^{m} \delta_{n}(j, \alpha) a_{j, \alpha}^{n(j, \alpha)}(x) Q_{n-\frac{|\alpha|}{p}(x ; \partial) \partial^{\alpha}} \\
\text { for } n \geqq n_{0}+1
\end{array}\right.
$$

where $\delta_{n}(j, \alpha)=\sigma(j, n-n(j, \alpha)-1)$

$$
=\left\{\begin{array}{c}
1 \\
\frac{1}{\left(n-\frac{|\alpha|}{p}+j-1\right)\left(n-\frac{|\alpha|}{p}+j-2\right) \cdots\left(n-\frac{|\alpha|}{p}+1\right)} \\
(j=2, \ldots, m) .
\end{array}\right.
$$


We prove Lemma 1 by the induction. When $n=n_{0}$, it is clear. Suppose that Lemma 1 is valid for any $h ; n_{0} \leqq h \leqq n-1$. Then we have

$$
\left\{\begin{array}{l}
v_{n}(x)=\frac{1}{n} \sum_{j=1}^{m} \sum_{\alpha} \sum_{\mu=n(j, \alpha)}^{n-1} \sigma(j, n-\mu-1) a_{j, \alpha}^{\mu}(x) \partial^{\alpha} \\
=\frac{1}{n}\left[\sum_{j=1}^{m} \sum_{|\alpha|=p-j}(x ; \partial) f(x)+R_{n-\mu-j}(x ; \partial) f(x)\right] \\
\left.\quad(x ; \partial) \partial^{\alpha} f(x)\right]+\frac{1}{n}\left[\sum_{j=1|\alpha|<p(n(j, \alpha)+j)}^{m} \sigma(j, n-n(j, \alpha)-1) a_{j, \alpha}^{n(j, \alpha)}(x) Q_{n-n(j, \alpha)-j}\right. \\
\quad a_{j, \alpha}^{n(j, \alpha)}(x) Q_{n-n(j, \alpha)-j}(x ; \partial) \partial^{\alpha} f(x) \\
\left.+\sum_{j=1}^{m} \sum_{\alpha} \sum_{\mu>n(j, \alpha)} \sigma(j, n-\mu-1) a_{j, \alpha}^{\mu}(x) Q_{n-\mu-j}(x ; \partial) \partial^{\alpha} f(x)-1\right) \\
+\sum_{j=1}^{m} \sum_{\alpha} \sum_{\mu \geqq n(j, \alpha)} \sigma(j, n-\mu-1) a_{j, \alpha}^{\mu}(x) \\
\left.\quad\left(\partial^{\alpha} Q_{n-\mu-j}(x ; \partial)-Q_{n-\mu-j}(x ; \partial) \partial^{\alpha}+\partial^{\alpha} R_{n-\mu-j}(x ; \partial)\right) f(x)\right] \\
=Q_{n}(x ; \partial) f(x)+R_{n}(x ; \partial) f(x),
\end{array}\right.
$$

where

$$
\begin{array}{r}
Q_{n}(x ; \partial)=\frac{1}{n} \sum_{j=1|\alpha|=p(n(j, \alpha)+j)}^{m} \sigma(j, n-n(j, \alpha)-1) \\
a_{j, \alpha}^{n(j, \alpha)}(x) Q_{n-n(j, \alpha)-j}(x ; \partial) \partial^{\alpha} .
\end{array}
$$

It is easy to verify that $Q_{n}(x ; \partial)$ and $R_{n}(x ; \partial)$ satisfy the requirements of Lemma 1.

\section{Proof of Theorem}

Let $N_{j}=\operatorname{Max}\{|\alpha|-j ;|\alpha|=p(n(j, \alpha)+j)\}$ and $N=\operatorname{Max} N_{j}$. It is represented by $N \geqq 1$ that the modified principal part of $L^{j}$ has a term which does not belong to the kowalevskian part of $L$.

To prove the theorem, it suffices that if $N \geqq 1$, we can construct a right-hand side $f(x)$ such that the formal solution $\sum_{n} t^{n} v_{n}(x)$ for $f(x)$ does not converge in any neighbourhood of the origin. Let us show this 
fact.

We assume $N \geqq 1$ and let $j_{0}$ be the minimum number satisfying $N_{j}$ $=N$. We treat in the first place the case of one variable, and we see that the general case is reducible, in some sense, to that of one variable.

Case of one variable. We can put by Lemma 1,

$$
v_{n}(x)=\alpha_{n}(x) \partial^{p\left(n-n_{0}\right)} f(x)+R_{n}(x ; \partial) f(x) \quad \text { for } n \geqq n_{0},
$$

where $\left\{\alpha_{n}(x)\right\}$ satisfy the following

$$
\begin{cases}\alpha_{n 0}(x)=1, & \\ \alpha_{n}(x)=0 & \text { for } n: p \cdot n \text { non integer, } \\ \alpha_{n}(x)=\frac{1}{n} \sum_{j=1}^{m} \sum_{\alpha=p(n(j, \alpha)+j)} \delta_{n}(j, \alpha) a_{j, \alpha}^{n(j, \alpha)}(x) \alpha_{n-\frac{\alpha}{p}}(x) & \text { for } n: p \cdot n \text { integer. }\end{cases}
$$

From the assumption

$$
a_{j_{0}, j_{0}+N}^{\eta^{\left(j_{0}, j_{0}+N\right)}}(x) \not \equiv 0
$$

and at this time we may assume (see S. Mizohata [3] p. 225, [4])

$$
a_{j_{0}, j_{0}+N}^{\left(j_{0}, j_{0}+N\right)}(0) \neq 0
$$

Hereafter we denote briefly $\alpha_{n}=\alpha_{n}(0), a_{j, \alpha}=a_{j, \alpha}^{n(j, \alpha)}(0)$. (3.2) can be written (3.5) $\left\{\begin{array}{l}\alpha_{n_{0}}=1, \\ \alpha_{n}=0 \quad \text { for } n: p \cdot n \text { non integer, } \\ \alpha_{n}=\frac{1}{n} \sum_{j=1}^{m} \sum_{\alpha=p(n(j, \alpha)+j)} \delta_{n}(j, \alpha) a_{j, \alpha} \alpha_{n-\frac{\alpha}{p}} \quad \text { for } n: p \cdot n \text { integer. }\end{array}\right.$

Lemma 2. There exists an infinite sequence $\left\{\alpha_{n}\right\}$ satisfying

$$
\left|\alpha_{n}\right|>\frac{K^{n}}{\left(p\left(n-n_{0}\right)\right) !} \frac{p \cdot n}{2\left(j_{0}+N\right)} \mid
$$

where【】denotes the Gauss' sign, and $K$ some positive constant.

Proof. Let $v_{k}=k / p$, and 


$$
\beta_{k}= \begin{cases}v_{k} v_{k-1} \ldots v_{k_{1}} \alpha_{n} & \text { for } k=p \cdot n \\ 0 & \text { for } k<k_{1} \quad \text { or } k \neq p \cdot n \quad\left(k_{1}=p \cdot n_{0}\right)\end{cases}
$$

Then we have from (3.5) the recurrence formula for $\left\{\beta_{k}\right\}$ :

$$
\begin{cases}\beta_{k_{1}}=n_{0}, & \\ \beta_{k}=0 & \text { for } k \neq p \cdot n \\ \beta_{k}=\sum_{j=1}^{m} \sum_{\alpha=p(n(j, \alpha)+j)} \varepsilon_{k}(j, \alpha) a_{j, \alpha} \beta_{k-\alpha} & \text { for } k=p \cdot n\end{cases}
$$

where

$$
\varepsilon_{k}(j, \alpha)=\left\{\begin{array}{l}
v_{k-1} v_{k-2} \ldots v_{k-\alpha+1} \quad(j=1), \\
\frac{v_{k-1} v_{k-2} \ldots v_{k-\alpha+1}}{\left(v_{k-\alpha}+j-1\right)\left(v_{k-\alpha}+j-2\right) \ldots\left(v_{k-\alpha}+1\right)} \quad(j=2,3, \ldots, m) .
\end{array}\right.
$$

Lemma. If $N \geqq 1$ and that $n_{0}$ is chosen sufficiently large, then there exists an infinite subsequence $\left\{k_{r}\right\}$ of $\{k\}$ satisfyng

and

$$
\begin{aligned}
& \left|\beta_{k_{r+1}}\right| \geqq \sqrt{k_{r}}\left|\beta_{k_{r}}\right| \\
& k_{r+1}-k_{r} \leqq j_{0}+N .
\end{aligned}
$$

In fact, if $k_{r}$ is defined, then $k_{r+1}$ is defined as the minimum number $h\left(h>k_{r}\right)$ satisfying

$$
\left|\beta_{h}\right| \geqq \sqrt{k_{r}}\left|\beta_{k_{r}}\right|
$$

Accordingly, it suffices to prove the following fact:

If, posing briefly $k_{r}=k$,

$$
\left|\beta_{k+j}\right|<\sqrt{k}\left|\beta_{k}\right| \quad \text { for all } j: j=1,2, \ldots, j_{0}+N-1
$$

then

$$
\left|\beta_{k+j_{0}+N}\right| \geqq \sqrt{k}\left|\beta_{k}\right|
$$

We show that this fact is valid as follows: We have from (3.6) 


$$
\begin{aligned}
& \beta_{k+j_{0}+N}=\left[\varepsilon_{k+j_{0}+N}\left(j_{0}, j_{0}+N\right) a_{j_{0}, j_{0}+N} \beta_{k}\right] \\
& +\left[\sum_{\substack{j>j 0 \\
N_{j}=N}} \varepsilon_{k+j_{0}+N}(j, j+N) a_{j, j+N} \beta_{k+j_{0}-j}\right] \\
& +\left[\sum_{j<j_{0}} \sum_{\substack{\alpha \leq N_{j}+j \\
\alpha=p(\bar{n}(j, \alpha)+j)}} \varepsilon_{k+j_{0}+N}(j, \alpha) a_{j, \alpha} \beta_{k+j_{0}+N-\alpha}\right. \\
& +\sum_{\substack{\alpha<j 0+N \\
\alpha=p\left(n(j 0, \alpha)+j_{0}\right)}} \varepsilon_{k+j_{0}+N}\left(j_{0}, \alpha\right) a_{j_{0}, \alpha} \beta_{k+j_{0}+N-\alpha} \\
& \left.+\sum_{\substack{j>j 0 \\
N_{j} \leqq N}} \sum_{\substack{\left.\alpha<j_{0}+N \\
\alpha=p(n, \alpha)+j\right)}} \varepsilon_{k+j_{0}+N}(j, \alpha) a_{j, \alpha} \beta_{k+j_{0}+N-\alpha}\right] \\
& +\sum_{\substack{j>j_{0} \\
N_{j}=N}} \sum_{\substack{j 0+N \\
\alpha=p(n(j, \alpha)+j)}} \varepsilon_{k+j_{0}+N}(j, \alpha) a_{j, \alpha} \beta_{k+j_{0}+N-\alpha} \\
& \left.+\sum_{\substack{j>j o j \\
N_{j}<N<+N \leq p(n)=\alpha \leq j+N_{j}}} \sum_{k+j_{0}+N}(j, \alpha) a_{j, \alpha} \beta_{k+j_{0}+N-\alpha}\right] \\
& =A+B+C+D \text {. }
\end{aligned}
$$

Let us evaluate $A, B, C$ and $D$. Firstly, note the next facts:

(1) if $h \leqq k=k_{r}$, then $\left|\beta_{h}\right| \leqq\left|\beta_{k}\right|$

(2) for any $p(p>0)$, there exist constants $c(c>0), d(d>1)$

$$
c \leqq \prod_{i=1}^{s} \frac{v_{k+i+i_{0}}}{v_{k}+i+i_{0}} \leqq d \quad\left(s=1,2, \ldots, m, i_{0}=0,1,2, \ldots\right)
$$

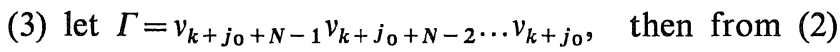

$$
c \cdot \Gamma \leqq \varepsilon_{k+j_{0}+N}(j, j+N) \leqq d \cdot \Gamma
$$

and

$$
\varepsilon_{k+j_{0}+N}(j, \alpha) \leqq \frac{d}{v_{k+j_{0}}} \cdot \Gamma \quad(\alpha \leqq j+N-1)
$$

We put

$$
\begin{aligned}
L & =\operatorname{Max}\left\{\left|a_{j, \alpha}\right| ; j>j_{0}, N_{j}=N, \alpha=j+N=p(n(j, \alpha)+j)\right\} \\
M & =\operatorname{Max}\left\{\left|a_{j, \alpha}\right| ; \alpha=p(n(j, \alpha)+j)\right\}
\end{aligned}
$$

Then we have 


$$
\begin{aligned}
& |A| \geqq c \Gamma\left|a_{j_{0}, j_{0}+N}\right|\left|\beta_{k}\right|, \\
& |B| \leqq d m L \Gamma\left|\beta_{k}\right|, \\
& |C| \leqq d m p(m+N) M \frac{\Gamma}{\sqrt{k}}\left|\beta_{k}\right|
\end{aligned}
$$

and

$$
|D| \leqq d m^{2} p M \frac{\Gamma}{k}\left|\beta_{k}\right|
$$

Therefore we have from (3.7)

$$
\left|\beta_{k+j_{0}+N}\right| \geqq \Gamma\left[c\left|a_{j_{0}, j_{0}+N}\right|-d m L-\frac{d m p(2 m+N)}{\sqrt{k}} M\right]\left|\beta_{k}\right|
$$

and, if necessary, choose $\lambda$ suitably and make the substitution

$$
t=\lambda \cdot \tau, \quad x=\lambda^{-\frac{1}{p}} \cdot y
$$

for $L$, we can suppose that

$$
c\left|a_{j_{0}, j_{0}+N}\right|-d m L>0 .
$$

Since $N \geqq 1$, we have $\Gamma \geqq v_{k+j_{0}} \geqq k / p$. Now we choose $k_{1}$ (i.e. $n_{0}$ ) so large that

$$
\frac{\Gamma}{\sqrt{k}}\left[c\left|a_{j_{0}, j_{0}+N}\right|-d m L-\frac{d m p(2 m+N)}{\sqrt{k}} M\right] \geqq 1 \quad \text { for } k \geqq k_{1}
$$

Hereafter we take $n_{0}\left(n_{0}=k_{1} / p\right)$ such a way. Consequently we obtain from (3.8) and (3.9),

$$
\left|\beta_{k+j_{0}+N}\right| \geqq \sqrt{k}\left|\beta_{k}\right| \quad \text { for } \quad k \geqq k_{1} .
$$

The proof of Lemma is complete.

Let us return to the proof of Lemma 2. From the Lemma, there exist constants $K_{0}$ and $K$ such that

$$
\left|\beta_{k_{r}}\right| \geqq K_{0}^{k_{r}}\left[\frac{k_{r}}{2\left(j_{0}+N\right)} \mid !\right.
$$


that is, for infinitely many $n$,

$$
\left|\alpha_{n}\right| \geqq \frac{K^{n}}{\left(p\left(n-n_{0}\right)\right) !} \frac{p \cdot n}{2\left(j_{0}+N\right)} \mid !
$$

This completes the proof of Lemma 2.

Now we return to the proof of the theorem. Let us construct $f(x)$. Let

$$
f(x)=\sum_{\substack{n \geq n_{0} \\ p n: \text { integer }}} e^{i \theta_{n}} x^{p\left(n-n_{0}\right)} \equiv \sum_{n}^{\prime} e^{i \theta_{n}} x^{p\left(n-n_{0}\right)}
$$

Then we have from (3.1)

$$
\begin{aligned}
v_{n}(0) & =\left.\left(\alpha_{n}(x) \partial^{p\left(n-n_{0}\right)}+R_{n}(x ; \partial)\right) f(x)\right|_{x=0} \\
& =\psi_{n}\left(\theta_{m} ; m<n\right)+e^{i \theta_{n}}\left(p\left(n-n_{0}\right)\right) ! \alpha_{n}
\end{aligned}
$$

where $\psi_{n}\left(\theta_{m} ; m<n\right)$ depends only on $\theta_{m}(m<n)$. We determine $\theta_{n}$ in such a way that

$$
\theta_{n}=\arg \psi_{n}\left(\theta_{m} ; m<n\right)-\arg \alpha_{n} \text { and } \theta_{n_{0}}=0 \text {. }
$$

For this $f(x)$, we have

$$
\left|v_{n}(0)\right| \geqq\left(p\left(n-n_{0}\right)\right) !\left|\alpha_{n}\right|
$$

and from Lemma 2

$$
\left|v_{n}(0)\right| \geqq K^{n}\left[\frac{p \cdot n}{2\left(j_{0}+N\right)} \mid !\right.
$$

for infinitely many $n$. This shows that the formal solution $\sum_{n} t^{n} v_{n}(x)$ for $f(x)$ can not converge in any neighbourhood of the origin.

General case. By the assumption and the definition of $N$ and $j_{0}$,

$$
\left[\sum_{|\alpha|=j_{0}+N} a_{j_{0}, \alpha}^{n\left(j_{0}, \alpha\right)}(x) t^{n\left(j_{0}, \alpha\right)} \partial^{\alpha}\right] \partial_{t}^{j_{0}}=t^{\frac{j_{0}+N}{p}-j_{0}}\left[\sum_{|\alpha|=j_{0}+N} a_{j_{0}, \alpha}^{n_{0}\left(j_{0}, \alpha\right)}(x) \partial^{\alpha}\right] \partial_{t}^{j^{0}} \not \equiv 0 .
$$

Hence, there exists $\xi=\left(\xi_{1}, \ldots, \xi_{l}\right) \neq 0$ such that

$$
\sum_{|\alpha|=j_{0}+N} a_{j 0, c^{\prime}}^{n\left(j_{0}, \alpha\right)}(x) \xi^{\alpha} \not \equiv 0
$$


and we proceed our argument under the assumption (see, S. Mizohata [3], [4])

$$
\sum_{|\alpha|=j_{0}+N} a_{j_{0}, \alpha}^{n_{j}\left(j_{0}, \alpha\right)}(0) \xi^{\alpha} \neq 0
$$

For the sake of simplicity, let $\xi_{1} \neq 0$ and make the substitution in $L$

$$
\begin{aligned}
& X_{1}=\xi_{1} x_{1}+\xi_{2} x_{2}+\cdots+\xi_{l} x_{l} \\
& X_{i}=x_{i} \quad(i=2,3, \ldots, l) .
\end{aligned}
$$

Then the modified weight $p, N$ and $j_{0}$ are invariant with respect to this change of variables and the modified principal part of $L$ is transformed into the modified principal part of the new operator and the term

$$
\sum_{|\alpha|=j_{0}+N} a_{j_{0}, \alpha}^{n\left(j_{0}, \alpha\right)}(x) \partial^{\alpha}
$$

is transformed into

$$
\left(\sum_{|\alpha|=j_{0}+N} a_{j_{0}, \alpha}^{n\left(j_{0}, \alpha\right)}(x) \xi^{\alpha}\right) \partial_{X_{1}}^{j_{0}+N}+R_{0}\left(X ; \partial_{X}\right)
$$

where $\underset{\partial_{X}}{\operatorname{order}} R_{0}\left(X ; \partial_{X}\right)=j_{0}+N$ and $\underset{\partial_{X_{1}}}{\operatorname{order}} R_{0}\left(X ; \partial_{X}\right)<j_{0}+N$.

If necessary, we make the above substitution and observing one of the variables $x_{i}$, for example $x_{1}$, we follow our reasoning in the case of one variable: If we adopt a function of $x: f(x)$ as

$$
f(x)=\sum_{n}^{\prime} e^{i \theta_{n}} x_{1}^{p\left(n-n_{0}\right)}
$$

then the formal solution $\sum_{n} t^{n} v_{n}(x)$ for $f(x)$ can not converge in any neighbourhood of the origin. This completes the proof of the theorem.

\section{References}

1] Hasegawa, Y., A Remark on the paper of S. Mizohata (in Japanese).

[2] Miyake, M., A Remark on Cauchy-Kowalevski's Theorem, Publ. RIMS Kyoto Univ. 10 (1974), 243-255.

[3] Mizohata, S., On kowalevskian systems, Uspehi Mat. Nauk. 29 (1974), 216-227.

[4] —, On Cauchy-Kowalevski's theorem. A necessary condition, in preparation. 